\title{
High-Energy Alerts in the Multi-Messenger Era
}

\author{
Daniela Dorner $^{1}\left(\mathbb{D}\right.$, Miguel Mostafá $2,3,4, * \mathbb{D}$ and Konstancja Satalecka ${ }^{5}$ (D) \\ 1 Department of Astronomy, Universität Würzburg, D-97074 Würzburg, Germany; \\ dorner@astro.uni-wuerzburg.de \\ 2 Department of Physics, Pennsylvania State University, University Park, PA 16802, USA \\ 3 Center for Multimessenger Astrophysics, Institute for Gravitation and the Cosmos, \\ Pennsylvania State University, University Park, PA 16802, USA \\ 4 Institut d'Astrophysique de Paris, 75014 Paris, France \\ 5 DESY, D-15738 Zeuthen, Germany; konstancja.satalecka@desy.de \\ * Correspondence: miguel@psu.edu
}

check for updates

Citation: Dorner, D.; Mostafá, M.; Satalecka, K. High-Energy Alerts in the Multi-Messenger Era. Universe 2021, 7, 393. https://doi.org/ 10.3390/universe7110393

Academic Editors: Ulisses Barres de Almeida, Michele Doro and Ezio Caroli

Received: 22 September 2021

Accepted: 15 October 2021

Published: 20 October 2021

Publisher's Note: MDPI stays neutral with regard to jurisdictional claims in published maps and institutional affiliations.

Copyright: (C) 2021 by the authors. Licensee MDPI, Basel, Switzerland. This article is an open access article distributed under the terms and conditions of the Creative Commons Attribution (CC BY) license (https:// creativecommons.org/licenses/by/ $4.0 /)$.

\begin{abstract}
The observation of electromagnetic counterparts to both high energy neutrinos and gravitational waves marked the beginning of a new era in astrophysics. The multi-messenger approach allows us to gain new insights into the most energetic events in the Universe such as gamma-ray bursts, supernovas, and black hole mergers. Real-time multi-messenger alerts are the key component of the observational strategies to unravel the transient signals expected from astrophysical sources. Focusing on the high-energy regime, we present a historical perspective of multi-messenger observations, the detectors and observational techniques used to study them, the status of the multimessenger alerts and the most significant results, together with an overview of the future prospects in the field.
\end{abstract}

Keywords: multi-messenger; real-time; high-energy; alerts

\section{Introduction}

The last century was marked by a fast evolution in our understanding of the Universe from the discovery of cosmic rays in 1912 to the first direct observation of gravitational waves in 2015. Particle physics and astrophysics went hand in hand towards discoveries of new particle species (antimatter, muons, pions, kaons, etc.) and the development of detection techniques (from cloud chambers to semiconductor detectors). Nowadays, not only photons but also charged particles (cosmic rays, CRs), neutrinos ( $v$ ), and gravitational waves (GWs) act as cosmic messengers and bring us news from distant corners of the Universe. We entered the era of multi-messenger astrophysics.

Very-high-energy $(\mathrm{VHE},>100 \mathrm{GeV}) \gamma$ rays play an important role in the multimessenger picture. They can be produced in both leptonic and hadronic interactions and, therefore, together with neutrinos, can reveal the localization and properties of the sources of CRs. They tell us about the highest particle energy (protons or electrons) reachable in these objects. The clues from their spectral shape reveal information about the environment (photon or matter fields) and the magnetic field strength both internal and external to the source. Recent discoveries of VHE $\gamma$-ray emission from Gamma Ray Bursts (GRBs) provide us with evidence for their connection to GW emitting objects.

In this review, we summarize the last 30 years of involvement of ground-based $\gamma$-ray telescopes in multi-messenger astrophysics, and give our perspective on the future developments in this field. We put a special emphasis on the follow-up of real-time alerts, which has already proven to be a very successful approach to study the multi-messenger universe.

\section{Historical Perspective}

The source 1ES1959+650, a near-by $(z=0.048)$ BL Lac type object, was the third extragalactic source discovered in VHE $\gamma$ rays [1]. Therefore, regular observations of 
this object were carried out, also in a form of coordinated multiwavelength campaigns, which was a novelty at the time. One of them, performed in 2002 by the Whipple and HEGRA VHE $\gamma$-ray instruments together with the Rossi X-Ray Timing Explorer, Boltwood and Abastumani optical observatories as well as VLA and UMRAO, brought a rather unexpected result. As reported in Ref. [2]: "Although the X-ray and $\gamma$-ray fluxes seemed to be correlated in general, we found an orphan $\gamma$-ray flare that was not accompanied by an X-ray flare." This short statement, indicating a departure from a purely leptonic emission scenario and a possibility of hadronic interactions at play [3] stirred the astro-particle community. This was particularly good news for the scientists looking for cosmic neutrino emissions with the AMANDA-II neutrino telescope. This small $\left(\sim 0.01 \mathrm{~km}^{3}\right)$ predecessor of IceCube had been recently completed (in 2000) and taking data. Soon, reports of five neutrino events observed from the direction of 1ES 1959+056, two of which arrived around the time of the orphan VHE $\gamma$-ray flare, followed $[4,5]$. Unfortunately, due to the trial factors arising from a posteriori choices of the time windows to be used for the statistical test, the significance of this coincidence cannot be properly estimated. Nevertheless, it proved the interest in simultaneous VHE $\gamma$-ray and neutrino observations-the idea for neutrinobased target-of-opportunity (ToO) triggers was born [6]. The other way around is also true. Real-time alerts of VHE $\gamma$-ray flares are of significant interest for the neutrino community.

While neutrino telescopes continuously monitor a large fraction of the sky, Imaging Air Cherenkov Telescopes (IACTs) are pointed instruments. For simultaneous observations, either a monitoring program or ToO observations are needed. As the sources of astrophysical neutrinos are not yet clearly identified, the ToO approach had to be chosen. One such program is the Gamma-ray Follow-Up (GFU), run since 2012 by IceCube [7] in collaboration with MAGIC, VERITAS, and H.E.S.S. [8]. The key idea is to identify neutrino source candidates among the $\gamma$-ray emitters and search in real-time for time-variable neutrino emission to reduce the overwhelming (six orders of magnitude) CR-induced background. In the current implementation, there are more than 330 monitored sources (mostly Active Galactic Nuclei, AGN) and the duration of the potential neutrino flare can vary from a few seconds to six months.

The joint work between the neutrino and electromagnetic (EM) observatories, as in [9] for example, demonstrated the power of a multi-messenger approach in astrophysics, and led to the concept of the Astrophysical Multimessenger Observatory Network (AMON) $[10,11]$. AMON is a continuous, real-time system designed to enable the discovery of the sources of transient multi-messenger signals by sifting through streams of sub-threshold events from several multi-messenger facilities, and correlating them in real-time in search of spatial and temporal coincidences. The first prototype of the AMON real-time system was built in 2013. The AMON server was designed to receive data (including sub-threshold events) from the member observatories, and to send alerts to the follow-up facilities when coincident signals were found. The prototype of the AMON server went online (i.e., started processing archival data) in July 2014, and the HTTPS client started running successfully at IceCube in August 2014. IceCube transmitted simulated neutrino events to AMON from August 2014 until February 2015, when AMON started receiving neutrino data from IceCube in real-time. This was the first real-time subthreshold data stream. The connection with the Gamma-ray Coordinates Network (GCN) was established in May 2015, and in August 2015, AMON started issuing real-time alerts via GCN to some collaborators (e.g., VERITAS and MASTER) for developing and testing their follow-up software and capabilities. By September 2015, archival data from Auger, ANTARES, IceCube, Swift-BAT, Fermi-LAT, and aLIGO (S6) were written to the AMON databases. AMON started the first coincidence analysis of archival data from participating neutrino (IceCube) and EM observatories (Fermi-LAT and Swift-BAT) in December 2015. The two high-uptime AMON production servers became fully operational in February 2016. These redundant servers were designed to have less than one hour of downtime per year. AMON started distributing in real-time the IceCube high-energy starting event 
(HESE) [12,13] and the extremely high energy (EHE) [12] alerts to the broader astrophysical community via GCN in April 2016.

\section{A New Multi-Messenger Era}

The first direct observation of gravitational waves from the merger of two black holes occurred on 14 September 2015, and it was announced by the LIGO/Virgo Collaboration (LVC) on 11 February 2016 [14]. Then, a GW event from the merger of two neutron stars was observed on 17 August 2017 [15], and the aftermath of this merger was seen across the electromagnetic spectrum [16]. This significant breakthrough in multi-messenger astrophysics clearly emphasized the urgency to establish a real-time alert system for GW events. The LVC started issuing public alerts through the GCN/TAN system from the beginning of the third observing run (O3) in April 2019. A significant percentage of O3 candidate events detected by LIGO were accompanied by corresponding triggers at Virgo. The values of the false alarm rate (FAR) were spread over a large range, with more than half of the events with a FAR greater than one per 20 years. The Kamioka Gravitational Wave Detector (KAGRA) in Japan became operational on February 25, 2020. However, KAGRA does not report their signals in real-time as LIGO and Virgo do. The O3 run (and therefore the GW alerts) ended earlier than scheduled on 27 March 2020 due to the COVID-19 pandemic.

\section{Paving the Way}

\subsection{Ground-Based Gamma-Ray Telescopes}

IACTs are pointed instruments with a field of view (FoV) of $\sim 3^{\circ}$ to $5^{\circ}$. Therefore, when receiving alerts, they require accurate information on the arrival direction of the multi-messenger events. Fast repositioning is an important feature of these instruments to be able to follow-up neutrino and other real-time alerts.

An example of the IACT adaptation for rapid follow-up are the MAGIC Telescopes. They were designed to detect VHE $\gamma$-ray emission from GRBs. Therefore, they were built with an extremely light-weight structure to allow for fast telescope movements. The system for repositioning and tracking sources was designed for rapid follow-up and has been equipped with calibration devices to track possible deformations of the structure [17]. Based on the fast repositioning, an automatic follow-up procedure was set up [18]. This 'GRB mode' needs to be enabled at the beginning of the night. To ensure the safety of the shift crew, there are switches that stop the motion of the telescopes when the door on the fence around the telescopes is opened. If an alert occurs when the 'GRB mode' is enabled, the automatic alert system takes control over all the subsystems to point to the position of the alert and start data taking as quickly as possible. Sub-minute times have been achieved from receiving the alert to taking data $[19,20]$.

Other IACTs have also implemented similar automatic follow-up systems, for example, [21,22]. For example, the H.E.S.S. collaboration constructed the largest IACT in the world to obtain the lowest energy threshold, which is important for these extragalactic observations. The large size is combined with rapid slewing capabilities ( $<1 \mathrm{~min}$ anywhere on the sky) and the deployment of a fully automatic alert system [21].

Although these automatic follow-up procedures were originally designed for GRB alerts, it is possible to adapt them to respond to neutrino and GW alerts, for example, [23].

\subsection{Neutrino Telescopes}

The largest $\left(1 \mathrm{~km}^{3}\right)$ operating neutrino observatory is IceCube [24], located at the South Pole. Two smaller detectors-ANTARES $\left(\sim 0.01 \mathrm{~km}^{3}\right)$ [25] and Baikal $\left(\sim 0.4 \mathrm{~km}^{3}\right)$ [26]-are immersed in the Mediterranean Sea and Lake Baikal, respectively. All of them detect neutrinos using the Cherenkov radiation from the secondary particles (muon, electron, tau, and hadronic showers) that are produced in neutrino interactions in water or ice. Neutrino telescopes use the bulk of the Earth as a shield against the atmospheric particle background. Therefore, they are most sensitive to the sources located on the hemisphere 
opposite to their location (e.g., the Northern hemisphere in the case of IceCube). The first astrophysical neutrino events were discovered with IceCube in 2013 [13], and more than 100 astrophysical candidates have been detected so far [27]. Although several types of searches for neutrino sources have been performed, including correlation studies with different sources catalogs [28,29] as well as blind searches in space and time [30,31], no unambiguous identification of a neutrino source has been made at a discovery level. The most significant excess ( $4.2 \sigma$ pre-trials) in the IceCube 10-year exposure map is at the location of the starburst galaxy NGC 1068. No VHE $\gamma$-ray excess has been detected from this source, despite a deep observation campaign performed by MAGIC [32].

The first neutrino events identified as astrophysical in origin, the High Energy Starting Events (HESE), were mostly of the cascade topology. This made the estimation of the arrival direction difficult, especially in ice, where the scattering length is shorter than in water. HESE events have localization uncertainties of the order of hundreds of square degrees. Due to the improvements in background rejection and event reconstruction, the diffuse astrophysical neutrino flux was also measured with track-like events; a.k.a. Extremely High Energy (EHE) events [33]. The localization uncertainty of EHE events was reduced to a few square degrees, that is, an area comparable to the typical field of view of an IACT. The experience gained with these two streams and the advances in the online event reconstruction allowed IceCube to establish the first public real-time neutrino channels: the EHE and HESE alerts. The IceCube collaboration issued the first real-time alert (EHE-160424A) on April 24, 2016, and it was followed up by all IACTs [34].

\subsection{The GCN/TAN System}

As the duration of GRBs is of the order of $<2(\sim 10-100)$ s for short (long) bursts, followup observations must rely on fast communications. For this reason, the GRB Coordinates Network (GCN)/Transient Astronomy Network (TAN) was started in 1992 [35]. The GCN/TAN system currently distributes the locations of GRBs and other transients (in the Notices) and also the reports (called Circulars) of the follow-up observations. The GCN/TAN Notices consist of the real-time (and near real-time due to telemetry downlink delays) distribution of GRB/transient locations (including images, spectra, and light curves) detected by various spacecrafts such as Swift, Fermi, MAXI, INTEGRAL, IPN, $M O A$ (gravitational lensing events), and others. The latency for missions with real-time downlinks is in the 2-10 s range. The GCN/TAN Circulars are natural language, prosestyle messages (as opposed to the "TOKEN: value" style of the Notices, easily readable by a machine) from follow-up observers (ground-based and space-based optical, radio, X-ray, $\mathrm{TeV}$, and other particle observers) reporting on their results (detections or upper limits). The GCN/TAN System is a very important tool in the context of multi-messenger astrophysics for the communication of alerts and the coordination of follow-up observations.

Looking forward, the Time-domain Astronomy Coordination Hub (TACH) [36] is a new effort to expand upon the GCN/TAN system. TACH will add new capabilities to GCN to enable community coordination of follow-up observations including improved user configuration flexibility, output protocols, reliability, speed, and cross-correlation between missions. TACH will also provide the infrastructure for joint $\gamma$-ray mission localizations in an open source platform, which will be especially relevant for the upcoming generation of GRB satellites.

\subsection{ATels}

Discoveries of supernovae and other transient objects are usually made public via Astronomer's Telegrams (ATels [37]). ATels are short web-based notifications commonly used to report and comment upon new astronomical observations of transient sources and to eventually trigger follow-up observations. The prompt nature of the ATels facilitates the distribution of observational results in the context of an unfolding transient astrophysical event. In many cases, the triggering observation has more information, for example, the full light curve, which is not included in the original ATel. Besides the daily emails with the 
selected areas of interest, readers may request the Instant Email Notices, which are used to report the discovery of one of several different types of objects, as well as new outbursts of previously known transients. The Instant Email Notices are sent immediately upon receipt.

Both GCN and ATels are widely used by all IACTs to coordinate their observations and to announce source discoveries or alert follow-up results.

\section{Current Situation}

In this section, we present the state of the alerts from the point of view of $\gamma$-ray telescopes. We first describe the alerts that are being sent from/within the $\gamma$-ray community. We then introduce the alerts that are being sent to the $\gamma$-ray community both from individual-messenger channels (either through AMON or direct-communication channels) and from multi-messenger real-time coincidence analyses. Finally, we discuss how IACTs receive and react to these alerts.

\subsection{Sending Alerts}

The $\gamma$-ray community communicates alerts very actively in the context of transients and coordination of multiwavelength follow-ups for interesting events such as AGN flares. For those events and based on a memorandum of understanding (MoU), the $\gamma$-ray instruments such as FACT, Fermi, HAWC, H.E.S.S., MAGIC, and VERITAS exchange information directly. Different trigger thresholds are set depending on the brightness of the sources. These alerts are communicated via email. In the case of events with a wider range of interest like the detection of a new $\gamma$-ray source or an exceptional flaring activity, an additional ATel is issued. (ATels are also used to request multiwavelength observations.) As described in Section 3.3, the GCN Circulars are the method of communication for follow-up observations or upper limits in the case of non-detections.

In addition to the emails under the MoU, GCN Circulars, and ATels, FACT informs all interested astronomers about flaring activities of blazars. A trigger threshold of three times the flux of the Crab Nebula (Crab Unit, CU) at TeV energies is used for the brightest blazars: Mrk 421 and Mrk 501, while for other blazars 0.5 CU is used [38]. Additionally, these alerts are also sent to AMON. As the correlation of sub-threshold events is an important feature of AMON, more elaborate trigger thresholds and algorithms are under investigation [39].

The HAWC collaboration sends two types of alerts under the MoU. The first alert stream is an online flare monitor that is based on the detection of variability using Bayesian blocks $[40,41]$. This stream is comprised of four channels that are used for different source classes. The alerts are issued with a FAR of 7.5 events per year for each channel, resulting in a total FAR of 30 events per year. One channel is dedicated to Mrk 421 and Mrk 501, and a second channel is used for the rest of the known TeV sources. The other two channels are for sources from the Fermi-LAT 2FHL catalog [42], one for nearby sources (with known redshift smaller than 0.3) and the other one for the rest of the sources in that catalogue. The second alert stream from HAWC is based on significant excesses ( $>5 \sigma$ post trials) in the all-sky map in different time scales $(0.5,1,2$ and 3 transits across the field of view of HAWC) [43]. These alerts are sent by email to MoU partners using different, pre-established FARs.

IACTs are follow-up instruments in general. They can produced their own alerts though. For example, an AGN that flares while is being observed can trigger an alert. In any case, there are no alerts being produced automatically as it can be done with an instrument with a high duty cycle like HAWC.

\subsection{AMON Pass-Through Alerts}

Among the data constantly being sent to AMON, there are also single-messenger events, that the triggering observatories want to distribute to the community in real time. In these cases, AMON passes the event information as an alert to enable follow-up observations in real time. An example of the importance of these pass-through channels is the event IceCube-170922A [44] discussed below in Section 5.2. 


\subsubsection{IceCube Tracks}

AMON distributes alerts in real-time via GCN Notices containing the information about neutrino events observed with IceCube that are likely of astrophysical origin [33]. There are two pass-through streams from IceCube. The first one is based on track-like neutrino candidates. For each candidate event, the alert includes the localization in the sky (with the $50 \%$ and $90 \%$ uncertainty radii), an estimate of the neutrino energy, a likelihood that the event is an astrophysical neutrino (signalness), and the corresponding FAR.

The IceCube collaboration has developed three event selections that result in these prompt high-energy track alerts. The Gamma-ray Follow-Up (GFU) track selection uses a machine learning algorithm that, based on the muon energy or the deposited charge, identifies coincidences of two or more tracks with a time window of up to three weeks [7]. These searches target sources from a predefined source catalog. Thus, GFU alerts are optimized for $\gamma$-ray follow-up as they are likely to be of astrophysical origin. The first GFU alert was sent in 2012. The HESE selection identifies high-energy neutrino events where the interaction vertex is contained within the instrumented volume [13]. The EHE track selection identifies $\mathrm{PeV}$ neutrinos. This selection was improved from the diffuse EHE neutrino search [45] by optimizing the requirements on the quality of the fit, the declination, and the total charge observed in the event (as a proxy for the neutrino energy). This modification increased the astrophysical purity of track-like candidates and the sensitivity to $\mathrm{PeV}$ neutrinos. These three selections combined provide a sample of likely-astrophysical track-like neutrino events, predominantly arising from muon neutrino charge-current interactions. The median angular resolution of these events is energy-dependent, but is better than $0.25^{\circ}$ for neutrino energies above $200 \mathrm{TeV}$.

These alerts are further classified (since 2019) as either Gold or Bronze alerts depending on their average astrophysical purity ${ }^{1}$. Gold (Bronze) alerts are well-reconstructed, highenergy, track-like neutrino candidates with a signalness greater than $50 \%(30 \%)$.

The expected all-sky annual alert rate is 28 , where eight alerts are from astrophysical neutrino events and 20 from atmospheric backgrounds. These rates are consistent with the observed rates of alert-qualifying events in the 7-year archival IceCube data. These alerts are not expected to be uniformly distributed in declination because there is a zenith dependence of the atmospheric backgrounds and the distribution itself is energy dependent due to the absorption of high-energy neutrinos in the Earth's core. The declination distribution of these alerts is expected to peak just above the equator in the Northern hemisphere.

\subsubsection{IceCube Cascades}

The high-energy track events were the first real-time alerts from IceCube because of the good angular resolution and fast reconstruction of these events. In recent years, the selection and reconstruction of high-energy cascade events has also been improved (and made possible in near real time) by the use of two neural networks.

A first neural network [46] is used to select cascade events contained inside of the detector; that is, the cascade events in the HESE sample are the starting point for this alert stream. The inputs of this classifier are the digitization of the waveforms (charge vs. time) recorded at each IceCube optical module. The architecture of this deep neural network is described in detail in Refs. [46,47]. This improved event selection is able to better reject tracks. Thus, it reduces the amount of atmospheric muons and neutrinos and leads to $\sim 8$ events per year with an astrophysical purity larger than $85 \%$. It is important to mention that this event classifier does not make a selection based on angular resolution. However, follow-up observatories are able to select events based on the angular uncertainty (provided in the alerts) that best fit the capabilities of their instrument.

A second neural network [48], used for the event reconstruction, improves the angular resolution. It takes a few seconds to run (instead of about a day for the previous reconstruction), allowing the reconstruction of cascade events in real time. Similarly to the event classifier, the input of the reconstruction network consists of a three-dimensional grid approximating the detector, as well as two other grids for the lower and upper parts 
of DeepCore. These grids also have a fourth dimension containing the time and charge information for each optical module. This neural network outputs the direction and the energy of the incoming neutrino, and the uncertainties on both parameters. The improved reconstruction results in $50 \%$ of the events having an angular resolution (68\% containment) better than $7^{\circ}$, including systematic uncertainties. The $50 \%$ and $90 \%$ containment angular error radii, corresponding to a circularized error, are reported in the alerts. However, the neural network is able to compute more sophisticated error contours that can be asymmetrical. These contours are also reported in the alerts as FITS files containing the probability density of the neutrino direction.

Cascade events from the Southern sky have a larger astrophysical purity because atmospheric neutrinos can be rejected. Additionally, the number of cascade events from astrophysical origin is lower in the Northern sky because the Earth is not transparent to high-energy neutrinos. Thus, the probability that an event is of astrophysical origin that is reported in the alerts accounts for three bins in zenith distance. Alerts are produced for events with an astrophysical likelihood higher than 0.9 , which corresponds to a FAR of 0.311 events per year.

\subsubsection{HAWC GRB-like Triggers}

Another important pass-through stream is the one dedicated to alerts from short timescale excesses in HAWC data. HAWC monitors the multi-TeV sky with an instantaneous field of view of $2 \mathrm{sr}$ and a duty cycle greater than $90 \%$. These alerts contribute to the on-going searches for VHE emission from GRBs, and especially for multiwavelength and multi-messenger studies.

The search for excesses uses a fixed-width sliding time window over the $\sim 25 \mathrm{kHz}$ of air showers reconstructed online at the HAWC site. The spatial search is done up to $50^{\circ}$ in zenith distance (i.e., declination approx. between $-31^{\circ}$ and $69^{\circ}$ ), in $\sim 2^{\circ} \times 2^{\circ}$ square bins (in right ascension and declination), and for time windows of $0.2,1,10$ and $100 \mathrm{~s}$. In each time window, all points in the FoV are tested against the null hypothesis that the local air-shower count comes from the rate of cosmic rays that pass the background suppression $(\sim 500 \mathrm{~Hz})$. Significant upward fluctuations from the expected number of background counts are interpreted as candidate VHE photons from GRB emission.

The uncertainty in the position of the excess reported in the alerts was derived from Monte Carlo simulations. The $68 \%$ containment radius is between $0.4^{\circ}$ and $0.8^{\circ}$ depending on the number of background events.

The threshold for sending these alerts was set at one event per year. This high falsepositive threshold is due to the number of trials associated with searching the entire field of view for all time windows. The trials calculation takes into account the fact that the search algorithm compares probabilities from multiple bins to select the result that is least consistent with the null hypothesis. The search algorithm and, more importantly, the calculation of this post-search false positive rate are explained in more detail in Ref. [49].

\subsection{Gravitational Wave Alerts}

The LIGO Scientific Collaboration and the Virgo Collaboration jointly analyze their data in real time to detect and localize transients from compact binary mergers and other possible sources of gravitational waves. When a signal candidate is found, an alert is distributed through the GCN/TAN System to search for possible counterparts such as electromagnetic waves or neutrinos. It is important to note that the LVC does not use any broker, and the GW alerts are sent directly to GCN by the LVC.

Before the initial alert, there might be cases in which an Early Warning Notice may be issued up to tens of seconds before the merger. Early Warning alerts are rare, and only possible for exceptionally loud and nearby coalescence events. Then, a Preliminary Notice is issued automatically a few minutes after the GW candidate was detected. In both cases, the signal must have passed automated data quality checks, and there is no accompanying GCN Circular. Thus, these may be retracted later. An Initial Notice is issued 
only after human vetting, which is accompanied by a GCN Circular. If further analysis of the data results in improved estimates of the sky localization, significance, and/or event classification, an Update Notice is issued. There may be multiple updates for the same GW event, and these updates may be issued within hours, days, or even weeks after the event. As described in Section 4.5, further analysis may also conclude that the event is unlikely to be from an astrophysical origin. In these cases, the candidate is withdrawn and a Retraction Notice is issued.

GW alerts contain a unique identifier, a FAR, and a sky localization. The FAR quantifies the significance of the event, and the sky localization consists of the posterior probability distribution of the position of the source in the sky. Additionally, alerts for coalescence events contain information about the luminosity distance, and inferred source classification and properties.

The sources of GWs are localized in the sky using the observed time delays, amplitude and phase consistency of the GW signals at the different sites. Two interferometers can constrain the sky location to a broken annulus, and the presence of additional detectors in the network improves the localization further. For example, the average sky localization area $\left(90 \%\right.$ credible region) was $655 \mathrm{deg}^{2}$ for the eleven confident signals detected during $\mathrm{O} 1$ and $\mathrm{O} 2$, while the expected median for all types of binary systems during $\mathrm{O} 3$ with the Advanced LIGO and Virgo network is 300 $\mathrm{deg}^{2}$ [50].

\subsection{Multi-Messenger Alerts}

AMON receives and stores astrophysical data, searches for multi-messenger coincidences, and distributes electronic alerts for follow-up observations. As mentioned before, AMON issues two distinct types of alerts, the pass-through alerts described in the previous section in which AMON serves simply as a conduit for propagating the single-messenger event information, and a second type from the coincidence analyses of two or more data streams.

A coincidence analysis combines two or more data streams using a likelihood calculation to quantify the degree of correlation between different events. An FAR is determined from scrambled datasets to build a representative distribution of random coincidences, and a test statistics value is used to rank the coincidences. The FAR thresholds are verified using archival data [51]. Finally, the collaborations contributing the streams used in the coincidence analysis specify the thresholds for distributing the alerts.

\subsubsection{ANTARES-Fermi}

This alert is a real-time search for coincidences between $\gamma$ rays observed with Fermi-LAT and neutrinos detected with ANTARES. The ANTARES collaboration sends their neutrino data to AMON in real time over a private stream. Photon data from the LAT is downloaded, as it becomes publicly available on the LAT FTP server ${ }^{2}$. The LAT data are filtered to select photons with energies between $100 \mathrm{MeV}$ and $300 \mathrm{GeV}$, a LAT zenith angle of less than $90^{\circ}$, and arrival times within the boundaries of the good time intervals provided by the LAT satellite files. A $\gamma-v$ coincidence is defined as any photons arriving within $5^{\circ}$ and $1000 \mathrm{~s}$ of an ANTARES neutrino. A pseudo-log-likelihood test statistic (TS) is calculated for each coincidence, as described in Ref. [52]. This TS takes into account the point spread functions (PSF) of each photon and each neutrino, and the number of neutrinos and gamma rays in the coincidence. There is also a temporal weighting function for each neutrino and $\gamma$ ray in the coincidence that is equal to one for particles within $100 \mathrm{~s}$ of the (average) arrival time. Between $100 \mathrm{~s}$ and $1000 \mathrm{~s}$, this function scales inversely proportional to this time difference to allow for possible longer-timescale associations (as might result from low-luminosity GRBs, for example) while maintaining a preference for shorter-timescale associations. The TS also accounts for the $\gamma$-ray backgrounds for each photon at the coincidence location, and a factor (established by the ANTARES Collaboration for each individual neutrino candidate) that represents its likelihood for being of astrophysical origin. Alerts more significant than a 4/year threshold are sent out via GCN to the AMON follow-up partners. 
The latency of these alerts is temporarily of the order of one day because the ANTARES collaboration reviews manually the neutrino(s) that participate in the coincidence.

\subsubsection{IceCube-HAWC}

These AMON alerts are also based on the sub-threshold detections of $\gamma$ rays and neutrinos. More specifically, they are produced using data from the daily monitoring stream of the HAWC Observatory [49] and the track stream from IceCube [33]. The HAWC data consist of the daily excesses with a significance higher than $2.75 \sigma$. These so-called "hotspots" are locations in the sky with a cluster of events above the estimated cosmicray background level, and are identified online during a full transit of that sky location above the detector. Most of these events are expected to be background fluctuations. The IceCube data are generated by the online event selection (described in Section 4.2.1) and reconstruction algorithms that are tuned to select track-like, through-going neutrino events. It is also dominated by background events, which in this case are atmospheric neutrino events in the Northern hemisphere, and high-energy atmospheric muons in the Southern hemisphere.

The coincidences are defined by a temporal and spatial criteria. Namely, the selected neutrinos must be detected during the transit time of the HAWC hotspot, and its reconstructed arrival direction must be within a radius of $3.5^{\circ}$ from the HAWC hotspot localization. A ranking statistic, based on Fisher's method, is then calculated by combining the spatial uncertainties of the $\gamma$-ray and neutrino events, the probability of the HAWC event being compatible with a background fluctuation, the probability of seeing more than one neutrino from background in the HAWC transit period, and the probability of measuring such an energy (or higher) for the IceCube event assuming it is a background event. The uncertainty on the best position of the coincidences is $\mathcal{O}\left(0.2^{\circ}\right)$, because it is dominated by the uncertainty on the location of the $\gamma$-ray excesses. The alerts include the $50 \%$ and $90 \%$ containment radii around the best position of the coincidence. The FARs were determined from scrambled datasets. The reported FARs are derived for each coincidence based on the ranking statistic described in detail in Ref. [53]. The rate of alerts being sent to GCN is four per year. The latency of these alerts could be about $6 \mathrm{~h}$ because the location of the hotspot has to transit above the HAWC detector before the analysis can start. The coincidence analysis in the AMON servers takes less than a minute to run after the sub-threshold triggers are received.

\subsection{Offline Updates, Revisions of Alerts}

The real-time distribution of transient events is possible through rapid computations on-site (in space, at the South Pole, etc.) without human intervention. Thus, it may occur that an offline, more precise and more time-consuming reconstruction differs from the original information in the notice. In these cases, either a correction or a retraction is issued. For example, if a high energy neutrino is no longer identified as astrophysical, then a retraction notice is issued. Similarly, the automated on-board Fermi flight software generates GRB position notifications in real time. But if the ground-based, human-involved processing results in a revised position, the updated information is transmitted within a few hours after the burst. This is a feature common to all the alert streams that is crucial to conduct efficient follow-up observations.

\subsection{Receiving Alerts, Follow-Ups}

The ground-based $\gamma$-ray telescopes receive the various alerts by GCN, VOEvent and/or email. Details on the follow-up programs of the current generation of IACTs are described in $[7,34,44,54-58]$. In the initial stages, follow-up observations were manually scheduled. Currently, if an alert arrives during a dark night, IACT telescopes use a fast, automatic re-pointing that allows for an immediate observation. In the case of full Moon or bad weather, the observations are sometimes postponed for up to a few days after the alert and then scheduled manually. 
A recent report discusses the single-neutrino alerts that have been followed up by IACTs since October 2017 [8]. No detection of VHE $\gamma$-ray emission was announced in connection to a single neutrino alert. No change in the source emission was observed following-up a flare detected within the GFU program.

H.E.S.S. is the only operating IACT located in the Southern hemisphere, and the ANTARES neutrino detector also obtains the best sensitivity in the Southern sky. Therefore, the two experiments complement each other very well. Their alert systems are linked directly using a VOEvent protocol [59,60], which allows for a rapid exchange of alerts between the two experiments. So far H.E.S.S. has reported follow-up observations of two ANTARES alerts, one of them (ANT170130) with a record response time of $32 \mathrm{~s}$ after the neutrino alert [61]. This work highlights the synergy between KM3NeT and CTA South, and will facilitate the implementation of an early system for mutual real-time follow-up observations, and exchanges of data such as flares, spectral/angular shapes, and so forth.

In the early phase of GW searches (corresponding to the $\mathrm{O} 1$ and $\mathrm{O} 2$ runs), the alerts were private; that is, GCN Notices were sent only to those observers that had an MoU with the LVC. Notices were made public after the LVC published the corresponding GW events. Ad hoc follow-up observations during the $\mathrm{O} 1$ and $\mathrm{O} 2$ interval include the $\mathrm{BH}-\mathrm{BH}$ merger GW151226 [62] by MAGIC [63] and GW170104 [64] by VERITAS ${ }^{3}$. The first complete scans of the uncertainty regions were possible during $\mathrm{O} 2$ when $\mathrm{GW}$ events started being detected by all three interferometers. For example, the binary black hole merger GW170814 [65] was followed up by H.E.S.S. ${ }^{4}$

Although during $\mathrm{O} 3$ the Notices were immediately publicly distributed without the need of an MoU, the issue of the large localization maps remained. Thus, dedicated follow-up procedures needed to be devised. Examples of these procedures include the MAGIC Automatic Alert System [66] and the H.E.S.S. automatic GW follow-up chain [23]. This algorithm was optimized to initiate GW follow-up observations within less than 1 min after receiving the alert. As a consequence, H.E.S.S. observed six GW events out of the 67 non-retracted GW events detected during the first three observation runs of LIGO and Virgo.

\section{Highlight Results}

Multi-messenger observations allow us to exploit the synergies that are inherent in the signals emitted from cosmic sources via the electromagnetic, gravitational wave, highenergy neutrino, and cosmic-ray channels. The candidate sources expected to emit in two or more of these messengers include active galactic nuclei, gamma-ray bursts, supernovae, white dwarfs, and neutron stars. The EM counterparts have already made possible the first identification of the source of a binary neutron star (BNS) merger and have provided evidence of an association between a high-energy neutrino and a gamma-ray flare from a blazar. More importantly, we think that multi-messenger observations have the potential to illuminate questions of fundamental physics and to provide unique measurements and independent insights that will completely revolutionize our understanding of highenergy astrophysics. The results that we highlight in this section emphasize the need for a systematic approach to move forward from the "pioneering" phase into the "expansion" stage, where Multi-Messenger Astrophysics becomes a field of its own.

\subsection{Gamma-Ray Bursts}

GRBs are the most luminous explosions in our Universe. Their energy release in $X$ rays and $\gamma$ rays can be $\sim 10^{51-54} \mathrm{erg}$ in a matter of seconds (assuming that GRBs radiate isotropically) [67]. Known since the 1970's and observed at a rate of $\sim 1 /$ day by X-ray satellites, GRBs were the reason behind the implementation of automatic alert follow-up systems by the IACTs. As described in Section 3.1, the process evolved through several stages. For example, MAGIC obtained light-weighted, fast moving telescopes from a dedicated hardware design. Trigger and software solutions also lowered the energy threshold, and allowed for detection of distant sources up to redshifts of $\sim 1.0$ [68-70]. 
These factors and the observation strategies honed for more than a decade resulted in the discovery of GRB 190114C by MAGIC $[19,71]$ and the detection of GRB 180720B afterglow by H.E.S.S. [72]. The former was obtained with observations starting as fast as $52 \mathrm{~s}$ after the trigger. The latter came from observations $\sim 11 \mathrm{~h}$ after the trigger. These two significant results demonstrate how the differentiation of the follow-up strategies allow us to study the temporal evolution of transient events. Both observations confirmed that the $\gamma$-ray emission from GRBs can reach up to TeV energies and revealed that this component is as powerful as the already known low-energy synchrotron component.

Since those breakthrough results, two more detections were announced: GRB 190829A by H.E.S.S. [73], and GRB 201216C by MAGIC ${ }^{5}$. The H.E.S.S. observations of the former were performed on three consecutive nights, between $4 \mathrm{hrs}$ and $56 \mathrm{hrs}$ after the trigger. The similar characteristics in the X-ray and $\gamma$-ray bands proves difficult to describe the emission with simple one-zone models. The latter, at $z=1.1$, is the most distant source detected by an IACTs so far. All the discussed detections concern long GRBs, whose precursors are Supernova explosions. So far only hints of VHE $\gamma$-ray emission from a short GRB have been reported for GRB 160821B [20]. Short GRBs are particularly interesting due to their connection to GW-emitting BNS mergers. For a more detailed discussion of GRBs and the role of IACT follow-up observations, we refer the reader to another review in this issue [74].

\subsection{TXS 0506+056-Neutrino Blazar?}

On 22 September 2017, a high-energy track event was detected by the IceCube realtime alert system. An automated alert ${ }^{6}$ was distributed to the community within less than a minute, including an initial estimate of the arrival direction and energy $(\sim 120 \mathrm{TeV})$ of the event. Subsequent multiwavelength EM observations revealed a high probability coincidence with the known blazar TXS0506+056, which was flaring in $\gamma$ rays at that time [44]. The chance coincidence for the correlation was excluded at a confidence level of $99.73 \%$, making this the most significant association between a HE neutrino and an astrophysical $\gamma$-ray source up to date.

The H.E.S.S. telescopes were the first IACT on target with the follow-up delay of only $4 \mathrm{~h}$ with respect to the neutrino event arrival time. No significant $\gamma$-ray emission was detected [75]. VHE $\gamma$-ray emission from this source was discovered with the MAGIC telescopes thanks to the low-energy threshold and persistent observation strategy [76,77], and later confirmed by the VERITAS observatory [78]. IACT observations during this flaring period show TXS 0506+056 as a highly variable VHE $\gamma$-ray emitter [54].

Various theoretical explanations followed these exciting results [9,79-81]. Among others, the MAGIC Collaboration explored the multiwavelength broad-band emission from this source, and the connection of the observed $\gamma$ rays with the observed neutrino and cosmic rays in the jets of the AGN [77]. In fact, this work shows numerical evidence that TXS 0506+056 is able to accelerate CRs up to energies of $\sim 10^{18} \mathrm{eV}$. The assumptions on the geometrical structure of the TXS 0506+056 jets, which were made to explain the neutrino emission, were confirmed with $15 \mathrm{GHz}$ very long baseline interferometry (VLBI) imaging [82].

A dedicated study of past IceCube observations of this object revealed another highly significant period of neutrino activity in 2014-2015, establishing it as a first potential source of cosmic neutrinos [83]. It is worth mentioning that this flare did not trigger a GFU alert only because the source redshift was unknown at that time [84] and the GFU program relies on sources with known redshift. TXS 0506+056 was added to the source list during the GFU upgrade in 2019.

Unfortunately, there is very little multiwavelength data, in particular no VHE $\gamma$-ray data, for the 2014-2015 neutrino flaring period. IACTs started to regularly monitor this source after the neutrino alert of 2017. (VERITAS observed TXS 0506+056 in 2016-2017; that is, prior to IC170922A, with no detection.) Both MAGIC and VERITAS, together with multiwavelength partners, perform unbiased monitoring observations to study the 
long term behavior of TXS 0506+056 [85,86]. Open questions include the object's nature (FSRQ vs BL Lac [87]) and its duty cycle, which is an important ingredient to derive the coincidence probability between the different messengers.

\subsection{GW170817}

The detection of the first BNS system GW170817 [15] marked the start of a new era of multi-messenger astrophysics [16]. The multi-year follow-up campaigns of this single event enabled observations across the entire EM spectrum that confirmed that the merger of two neutron stars is able to power high-energy transients, such as short GRBs [88] and kilonovae. The H.E.S.S. observations of this event started on 17 August 2017 at 17:59 UTC, only $\sim 5$ min after the localization map was made public by the LVC, and $5.3 \mathrm{~h}$ after the original GW170817 alert. They found no significant $\gamma$-ray emission. Their upper limits on the VHE $\gamma$-ray flux constrained for the first time the non-thermal, high-energy emission following a BNS merger [89]. H.E.S.S. also performed a long-term campaign (between 124 and 272 days after the GW event) on the remnant of the BNS merger, covering the maximum in the X-ray emission. They derived limits on the magnetic field of the remnant in the context of different source scenarios [90].

Results from this single multi-messenger event clearly demonstrate the potential of multi-messenger astrophysics. This event also highlights the need for population studies to disentangle the physics at the source and the interactions in the environment.

\section{Discussion}

The multi-messenger detections in recent years have produced an enormous leap forward in our understanding of highly energetic, transient events. On the other hand, the observation of astrophysical sources via non-electromagnetic messengers has faced equally enormous challenges. New solutions are needed to meet the increased demand for low-latency analyses, and reach the promised greater rewards in the years ahead.

\subsection{Real-Time Tools, Alerts, and Follow-Up Campaigns}

Multi-messenger astrophysics relies heavily on sharing alerts in real time. In turn, these alerts have to be prioritized by each experiment considering their features, capabilities, and scientific objectives. It would be ideal to develop a set of common tools to identify potential sources of GWs and high energy neutrinos, and a community-wide coordination to maximize the number of multi-messenger observations. Only automated, systematic campaigns will guarantee unbiased coincidences and an optimized use of wide-field, allsky survey instruments and target of opportunity observations with narrow field of view telescopes. Given the number of alerts expected from the next generation of neutrino, GW, and electromagnetic observatories, this automatization of the observing campaigns has become a requirement.

Although many resources for EM counterpart identification exist, such as source catalogues and archival repositories (e.g., $\mathrm{ASDC}^{7}$, OpenUniverse ${ }^{8}, \mathrm{VO}^{9}$ ), they are still not fully connected to the real-time alert brokers. The first steps toward this connection are starting to be realized. One example is the novel tool called AstroCOLIBRI [91] that collects in one place the real-time alerts and the relevant information about the persistent and transient sources in the vicinity of the event. There is still some work to be done to fully integrate the natural language resources (e.g., GCN Notices and ATels) into the system.

It is also clear that, thanks to the increasing number of more sensitive telescopes that are planned or under construction (e.g., Vera Rubin Observatory, SKA), astronomy is entering the era of Big Data. Processing large amount of information very fast will be crucial to enable immediate follow-up of potential counterparts.

\subsection{The Big Data Challenge and Real-Time Analyses}

On the receiver end, efficient observation routines need to be implemented. This is especially important for pointing instruments that face hard choices such as: where to 
point? For how long? Is the origin of dark matter more important topic than GW follow-up? It is important to solve these issues before the next generation of experiments bring us an order of magnitude more real-time alerts.

In fact, the difficulties do not stop with an established multi-messenger coincidence. To estimate the significance of such coincidences, we will need data on the long-term behavior of the sources or reliable statistics of the transient occurrences. Currently, only a small number of VHE $\gamma$-ray emitters are monitored regularly and most of them are nearby blazars, for example, [92]. Moreover the monitoring is often biased towards flaring events, which prevents a proper estimation of the emission duty cycle.

\section{Outlook}

\subsection{Multi-Messenger Alerts}

There are several AMON coincidence alert streams under development. Similarly to the channel described in Section 4.4.2, AMON is in the final stages to start issuing alerts using sub-threshold detections of $\gamma$ rays from HAWC and neutrinos from ANTARES [93]. The AMON Team is working to generate and distribute low-latency alerts from the realtime coincidence analysis between Fermi-LAT data and the sub-threshold stream from IceCube. The archival analysis [94] showed hints of a possible correlation between the neutrino positions and persistently bright portions of the $\gamma$-ray sky. These AMON alerts will prompt rapid-response follow-up observations that could test a possible signature of $\gamma$-ray correlated structure in the high-energy neutrino sky. The AMON Team is also developing a correlation analysis using a new capability of the Swift Observatory that provides event-level data from the BAT on demand [95] to respond in real-time to transient triggers from sub-threshold GW events [96]. The goal of this coincidence stream is to identify short GRB-like emission with an arcminute localization for the sub-threshold GW triggers during O4. Finally, the AMON Team is developing an outlier detection method [97] to make a model independent combination of the sub-threshold data from multiple neutrino and gamma-ray experiments [98].

While cosmic rays have played a small role in real-time multi-messenger alerts, the Pierre Auger Collaboration is implementing a new data stream that will send to AMON ultra-high energy photon candidates. This stream will contain events with energies above $1 \mathrm{EeV}$ that satisfy a certain sub-threshold photon selection based on a Multivariate analysis [99].

\subsection{VHE $\gamma$ Rays}

The IACT community will also soon posses a new instrument to investigate the Universe at very high energies and with sensitivity an order of magnitude higher than the current telescopes generation. The future Cherenkov Telescope Array (CTA) [100] is under construction in two locations. CTA-North will be hosted at the Canary Island of La Palma (the same site as FACT and MAGIC) and CTA-South near Paranal in Chile. Both will consist of several telescopes, whose capacities are adapted to efficiently cover different energy ranges: 23-meter Large Size Telescope (LST), 12-meter Medium Size Telescope (MST) and 4-meter Small Size Telescope (STS). CTA has several performance characteristics which are important in the context of the follow-up of transient astrophysical messengers, like neutrinos and GWs. The first one is the ability to rapidly re-position to any location in the sky. For example the LSTs can re-position to anywhere in the sky above $30^{\circ}$ in just $20 \mathrm{~s}$. The second one is a large FoV. For LSTs it is $\sim 4^{\circ}$, for MSTs $\sim 8^{\circ}$ and for SSTs $\sim 10^{\circ}$. What is more, the CTA real-time analysis will search for transients in the whole CTA FoV and send alerts [101,102]. Therefore CTA will not only passively receive and follow-up alerts but be an active player among the multi-messenger observatories. CTA will explore the $\mathrm{GeV}-\mathrm{TeV}$ sky with a deeper sensitivity than previous IACT instruments. The larger field of view, the flexibility to map very large and arbitrary sky patches, and the rapid response time will make CTA an ideal instrument to detect possible VHE $\gamma$-ray counterparts to GW and neutrino events. 
The Large High Altitude Air Shower Observatory (LHAASO) has recently started operations and has already published about a dozen UHE cosmic accelerators in our galaxy [103]. Although LHAASO has also detected $\gamma$ rays with energies above $1 \mathrm{PeV}$ [104], their real-time alert system is under construction. In a longer time scale, the Southern Wide-field Gamma-ray Observatory (SWGO) will complement the existing and planned instruments to conduct these crucial observations in the Southern Hemisphere. SWGO will participate in archival analyses and in real-time searches for transients at different timescales covering a region of the sky not accessible to either HAWC or LHAASO [105].

\subsection{HE Neutrinos}

After ten years of data taking, the IceCube detector has started to see hints of neutrino emission from several sources, one of them being the famous blazar TXS 0506+056 [30]. However, those hints are at the level of $\sim 3$ standard deviations and do not yet reach the discovery level of $\geq 5$ standard deviations. In the next five years or so, neutrino astronomy will get a significant boost through the installation of several $\mathrm{km}^{3}$-scale facilities, such as Baikal-GVD [26] and KM3NeT [106]. By 2025, each one of these should have an effective detection volume comparable to the current IceCube observatory. A new initiative called the Planetary Neutrino Monitoring System (PLEvM [107]) has been proposed to move beyond the signal hints into source detections and neutrino astronomy. The goal is to combine the exposures of current and future neutrino telescopes distributed around the world. PLEVM will have full-sky coverage and will reach up to four times the exposure available today. On a longer timescale, an extension of the existing IceCube experiment, called IceCube-Gen2 [108], is planned to be deployed in the 2030 decade. The hybrid design, including radio and light-sensor arrays in ice and a surface array incorporated as a veto, will boost the discovery potential at the ultra-high energies ( $>100 \mathrm{PeV})$, not yet accessible to IceCube. On a similar timescale, the Pacific Ocean Neutrino Experiment (P-ONE [109]) will deploy a multi-cubic-kilometre neutrino telescope in the Pacific Ocean.

\subsection{Gravitational Waves}

On the GW front, the LIGO/Virgo/KAGRA collaboration is making good progress in preparation for $\mathrm{O} 4$. Although there is no definitive start date for $\mathrm{O} 4$ at the time of this writing, it is clear that $\mathrm{O} 4$ will not begin before August 2022. Assuming that there are no unexpected obstacles, the four-detector network is expected to achieve design sensitivity with a range of almost $200 \mathrm{Mpc}$ in O4. Further down the line, it is possible that the LIGOIndia detector may come online and become part of the international GW network at some point during $\mathrm{O} 5$.

As seen in the past observations, source localization using only timing for a two-site network yields an annulus on the sky. Adding the signal amplitude and phase resolves this to only parts of the annulus. However, even then sources are localized to regions of hundreds to thousands of square degrees $[110,111]$. For three detectors, the time delays restrict the source to two sky regions which are mirror images with respect to the plane passing through the three sites. Requiring consistent amplitudes and phase in all the detectors typically eliminates one of these regions [112]. Thus, this typically yields regions with areas of several tens to hundreds of square degrees. A large improvement of the localization capability is expected for $\mathrm{O} 4$, where the expanded network of detectors is also accompanied by higher sensitivities. With four (or more) interferometers, timing information alone is sufficient to localize to a single sky region, while the additional baselines help to localize within regions smaller than ten square degrees for some signals.

The LIGO/Virgo/KAGRA collaboration expects $~ 10$ detections of BNS mergers per year in $\mathrm{O} 4$. (For estimating the number of events expected to be detected in $\mathrm{O} 4$ they used an intermediate sensitivity curve for KAGRA and the target sensitivity curve for Advanced LIGO and for Advanced Virgo.) The median 90\% credible region for the localization area of BNS is $33 \mathrm{deg}^{2}$, while $38-44 \%(12-16 \%)$ of the events are expected to have a $90 \%$ credible region smaller than $20 \mathrm{deg}^{2}\left(5 \mathrm{deg}^{2}\right)$. Then, there are $\sim 80$ expected detections of binary 
black holes (BBH). The median $90 \%$ credible region for the localization area of $\mathrm{BBH}$ is $41 \mathrm{deg}^{2}$. Similarly, 35-39\% (11-14\%) of the BBH events are expected to have a $90 \%$ credible region smaller than $20 \mathrm{deg}^{2}\left(5 \mathrm{deg}^{2}\right)$ [50].

It is natural to expect even in the near future an increasing number of multi-messenger detections of binary mergers. This will make it possible to determine the equation of state of neutron stars, to probe the properties of different components of the ejected mass, to answer whether the BNS mergers are the primary channel of formation of heavy elements, and to understand the structure of the relativistic jets and the physics behind their formation. Also joint GW-neutrino observations with IceCube and KM3NeT may reveal coincident emissions of high-energy neutrinos from BNS mergers or other energetic astrophysical phenomena.

\subsection{Supporting Infrastructure}

Besides the current efforts to combine $\gamma$-ray data with the detections of gravitational waves and high-energy neutrinos, there are several initiatives to support and enhance multimessenger astrophysics. One example of these projects is the Scalable Cyberinfrastructure to support Multi-Messenger Astrophysics (SCiMMA) [113], which is funded by the U.S. National Science Foundation. The main goal of this community-wide project is to provide the necessary scalable cyberinfrastructure to support multi-messenger astrophysics by rapidly handling, combining, and analyzing the very large-scale distributed data from all the types of astrophysical measurements. Their proposed cyberinfrastructure will allow the community to take full advantage of the current facilities and also the next-generation projects for multi-messenger astrophysics.

Similarly, on the other side of the Atlantic the European Open Science Cloud (EOSC) is an open multi-disciplinary environment for hosting and processing research data to support EU science. The EOSC Future project plans to develop an environment with interoperable research data sets and other research outputs including publications and code, professional data services, and access to resources such as compute and storage and services like data discovery and archive. The EOSC Future will support European researchers in managing the entire lifecycle of data from sharing, managing and exploiting their own data to discovering, re-using and recombining the data sets of others [114].

Funding: M.M. acknowledges support from the U.S. National Science Foundation (NSF) under grants PHYS-1506232, PHY-1708146, PHY-1806854, and PHY-2110809, and from the Institute for Gravitation and the Cosmos (IGC) of the Pennsylvania State University. D.D. acknowledges the support of the German Bundesministerium für Bildung und Forschung (BMBF) and the Institut für Theoretische Physik und Astrophysik (ITPA). K.S. acknowledges the support of the European Union's Horizon 2020 Programme under the AHEAD2020 project (grant agreement n. 871158). Any opinions, findings, and conclusions or recommendations expressed in this material are those of the authors and do not necessarily reflect the views of NSF, IGC, BMBF, ITPA, or the Horizon 2020 Programme.

Data Availability Statement: Data sharing is not applicable to this article as no new data were created or analyzed in this study.

Acknowledgments: We would like to thank Fabian Schüssler and Marcos Santander for their careful reading of an early draft, and for their thoughtful comments that provided insight and expertise and helped us shape the paper.

Conflicts of Interest: The authors declare no conflict of interest. 


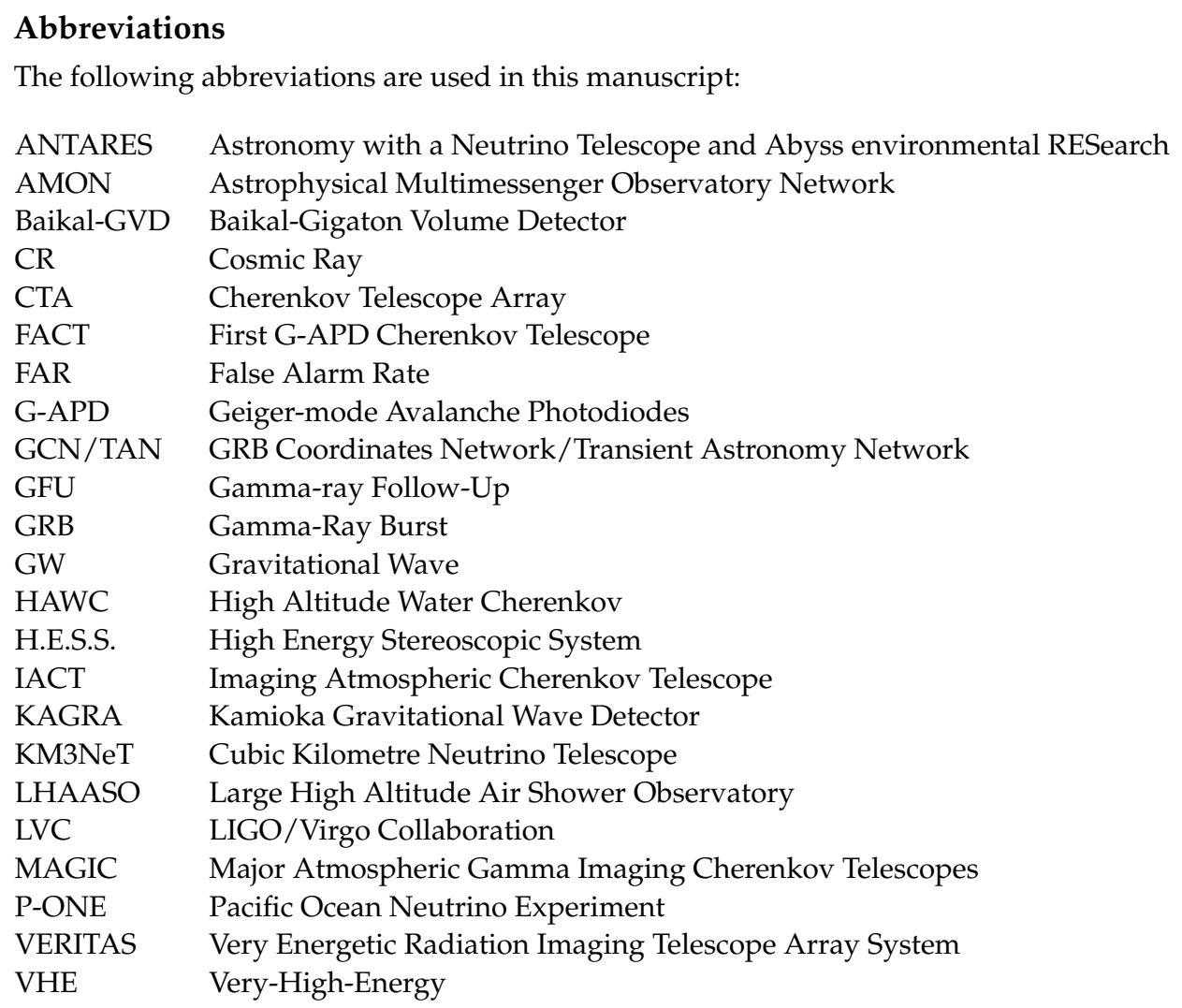

\section{Notes}

1 The GCN document describing the IceCube high-energy neutrino track alerts can be found at: https://gcn.gsfc.nasa.gov/doc/IceCube_High_Energy_Neutrino_Track_Alerts_v2.pdf (accessed on 19 October 2021).

2 The LAT photon data are located at: https://heasarc.gsfc.nasa.gov/FTP/fermi/data/lat/weekly/photon/ (accessed on 19 October 2021).

3 https://gcn.gsfc.nasa.gov/gcn3/21153.gcn3 (accessed on 19 October 2021).

https://gcn.gsfc.nasa.gov/gcn3/21673.gcn3 (accessed on 19 October 2021).

https:/ / gcn.gsfc.nasa.gov / other/201216C.gcn3 (accessed on 19 October 2021).

IceCube Collaboration, GRB Coordinates Network/AMON Notices 50579430_130033 (2017).

www.asdc.asi.it (accessed on 19 October 2021).

openuniverse.asi.it (accessed on 19 October 2021).

ivoa.net (accessed on 19 October 2021).

\section{References}

1. Nishiyama, T. Detection of a new TeV gamma-ray source of BL Lac object 1ES 1959+650. In Proceedings of the 26th International Cosmic Ray Conference (ICRC26), Salt Lake City, UT, USA, 17-25 August 1999; Volume 3, p. 370.

2. Krawczynski, H.; Hughes, S.B.; Horan, D.; Aharonian, F.; Aller, M.F.; Aller, H.; Boltwood, P.; Buckley, J.; Coppi, P.; Fossati, G.; et al. Multiwavelength Observations of Strong Flares from the TeV Blazar 1ES 1959+650. Astrophys. J. 2004, 601, 151-164. [CrossRef]

3. Böttcher, M. A Hadronic Synchrotron Mirror Model for the “Orphan” TeV Flare in 1ES 1959+650. Astrophys. J. 2005, 621, 176-180. [CrossRef]

4. Resconi, E. High energy neutrinos as cosmic messengers: AMANDA \& IceCube. In Proceedings of the Talk Given at the Aspen Winter Conference: The Highest Energy Physics, Aspen, CO, USA, 13-19 February 2005. Available online: http:// conferences.fn al.gov/aspen05/ (accessed on 19 October 2021).

5. Bernardini, E. Search for High Energy Astrophysical Neutrinos with the AMANDA Detector at the South Pole. In Proceedings of the Talk Given at the International Workshop on Particles and Radiation from Cosmic Accelerators, Chiba, Japan, 2-5 March 2005. Available online: http:/ / www.astro.phys.s.chiba-u.ac.jp/ca2005/ (accessed on 19 October 2021).

6. Bernardini, E. Multi-Messenger Studies with AMANDA/IceCube: Observations and Strategies. In Proceedings of the 7th Workshop towards a Network of Atmospheric Cherenkov Detectors, Palaiseau, France, 27-29 April 2005.

7. Murase, K.; Halzen, F.; Ahlers, M. Very high-energy gamma-ray follow-up program using neutrino triggers from IceCube. $J$. Instrum. 2016, 11, P11009. [CrossRef] 
8. Satalecka, K.; Bernardini, E.; Dorner, D.; Mezek, G.K.; Jin, W. Searching for VHE gamma-ray emission associated with IceCube neutrino alerts using FACT, H.E.S.S., MAGIC, and VERITAS. arXiv 2021, arXiv:2109.04350.

9. Keivani, A.; Murase, K.; Petropoulou, M.; Fox, D.B.; Cenko, S.B.; Chaty, S.; Coleiro, A.; DeLaunay, J.J.; Dimitrakoudis, S.; Evans, P.A.; et al. A Multimessenger Picture of the Flaring Blazar TXS 0506+056: implications for High-Energy Neutrino Emission and Cosmic Ray Acceleration. Astrophys. J. 2018, 864, 84. [CrossRef]

10. Smith, M.; Fox, D.; Cowen, D.; Mészáros, P.; Tešić, G.; Fixelle, J.; Bartos, I.; Sommers, P.; Ashtekar, A.; Babu, G.J.; et al. The Astrophysical Multimessenger Observatory Network (AMON). Astropart. Phys. 2013, 45, 56-70. [CrossRef]

11. Ayala Solares, H.A.; Coutu, S.; Cowen, D.; DeLaunay, J.J.; Fox, D.B.; Keivani, A.; Mostafá, M.; Murase, K.; Oikonomou, F.; Seglar-Arroyo, M.; et al. The Astrophysical Multimessenger Observatory Network (AMON): Performance and Science Program. Astropart. Phys. 2019, 114, 68-76. [CrossRef]

12. Aartsen, M.G.; Abbasi, R.; Abdou, Y.; Ackermann, M.; Adams, J.; Aguilar, J.A.; Ahlers, M.; Altmann, D.; Auffenberg, J.; Bai, X.; et al. First Observation of PeV-Energy Neutrinos with IceCube. Phys. Rev. Lett. 2013, 111, 021103. [CrossRef]

13. IceCube Collaboration. Evidence for High-Energy Extraterrestrial Neutrinos at the IceCube Detector. Science 2013, $342,1242856$. [CrossRef]

14. Abbott, B.P.; Abbott, R.; Abbott, T.D.; Abernathy, M.R.; Acernese, F.; Ackley, K.; Adams, C.; Adams, T.; Addesso, P.; Adhikari, R.X.; et al. Observation of Gravitational Waves from a Binary Black Hole Merger. Phys. Rev. Lett. 2016, 116, 061102. [CrossRef]

15. Abbott, B.P.; Abbott, R.; Abbott, T.D.; Acernese, F.; Ackley, K.; Adams, C.; Adams, T.; Addesso, P.; Adhikari, R.X.; Adya, V.B.; et al. GW170817: Observation of Gravitational Waves from a Binary Neutron Star Inspiral. Phys. Rev. Lett. 2017, 119, 161101. [CrossRef]

16. Abbott, B.P.; Bloemen, S.; Canizares, P.; Falcke, H.; Fender, R.P.; Ghosh, S.; Groot, P.; Hinderer, T.; Hörandel, J.R.; Jonker, P.G.; et al. Multi-messenger Observations of a Binary Neutron Star Merger. Astrophys. J. 2017, 848, L12. [CrossRef]

17. Bretz, T.; Dorner, D.; Wagner, R.M.; Sawallisch, P. The drive system of the major atmospheric gamma-ray imaging Cherenkov telescope. Astroparticle Physics 2009, 31, 92-101. [CrossRef]

18. Berti, A. Search for High Energy emission from GRBs with MAGIC. Proc. Int. Astron. Union 2016, 12, 70-73. [CrossRef]

19. MAGIC Collaboration. Teraelectronvolt emission from the $\gamma$-ray burst GRB 190114C. Nature 2019, 575, 455-458. [CrossRef]

20. Acciari, V.A.; Ansoldi, S.; Antonelli, L.A.; Engels, A.A.; Asano, K.; Baack, D.; Babić, A.; Baquero, A.; de Almeida, U.B.; Barrio, J.A.; et al. MAGIC Observations of the Nearby Short Gamma-Ray Burst GRB160821B. Astrophys. J. 2021, $908,90$. [CrossRef]

21. Aharonian, F.; Akhperjanian, A.G.; De Almeida, U.B.; Bazer-Bachi, A.R.; Behera, B.; Benbow, W.; Bernlöhr, K.; Boisson, C.; Bochow, A.; Borrel, V.; et al. HESS observations of bursts in 2003-2007. Astron. Astrophys. 2009, 495, 505-512. [CrossRef]

22. Acciari, V.A.; Aliu, E.; Arlen, T.; Aune, T.; Beilicke, M.; Benbow, W.; Bradbury, S.M.; Buckley, J.H.; Bugaev, V.; Byrum, K.; et al. Veritas observations of gamma-ray bursts detected byswift. Astrophys. J. 2011, 743, 62. [CrossRef]

23. Ashkar, H.; Brun, F.; Füßling, M.; Hoischen, C.; Ohm, S.; Prokoph, H.; Reichherzer, P.; Schüssler, F.; Seglar-Arroyo, M. The H.E.S.S. gravitational wave rapid follow-up program. J. Cosmol. Astropart. Phys. 2021, 2021, 045. [CrossRef]

24. Aartsen, M.G.; Ackermann, M.; Adams, J.; Aguilar, J.A.; Ahlers, M.; Ahrens, M.; Altmann, D.; Andeen, K.; Anderson, T.; Ansseau, I.; et al. The IceCube Neutrino Observatory: Instrumentation and online systems. J. Instrum. 2017, 12, P03012. [CrossRef]

25. Ageron, M.; Aguilar, J.A.; Al Samarai, I.; Albert, A.; Ameli, F.; André, M.; Anghinolfi, M.; Anton, G.; Anvar, S.; Ardid, M.; et al. ANTARES: The first undersea neutrino telescope. Nucl. Instrum. Methods Phys. Res. A 2011, 656, 11-38. [CrossRef]

26. Avrorin, A.D.; Avrorin, A.V.; Aynutdinov, V.M.; Bannash, R.; Belolaptikov, I.A.; Brudanin, V.B.; Budnev, N.M.; Domogatsky, G.V.; Doroshenko, A.A.; Dvornicky, R.; et al. Baikal-GVD: First results and prospects. In European Physical Journal Web of Conferences; EDP Sciences: Les Ulis, France, 2019; Volume 209, p. 01015. [CrossRef]

27. Abbasi, R.; Ackermann, M.; Adams, J.; Aguilar, J.A.; Ahlers, M.; Ahrens, M.; Alispach, C.; Alves, A.A., Jr.; Amin, N.M.; Andeen, K.; et al. IceCube high-energy starting event sample: Description and flux characterization with 7.5 years of data. Phys. Rev. D 2021, 104, 022002. [CrossRef]

28. Aartsen, M.G.; Abraham, K.; Ackermann, M.; Adams, J.; Aguilar, J.A.; Ahlers, M.; Ahrens, M.; Altmann, D.; Andeen, K.; Anderson, T.; et al. The Contribution of Fermi-2LAC Blazars to Diffuse TeV-PeV Neutrino Flux. Astrophys. J. 2017, 835, 45. [CrossRef]

29. Albert, A.; André, M.; Anghinolfi, M.; Ardid, M.; Aubert, J.J.; Aublin, J.; Avgitas, T.; Baret, B.; Barrios-Martí, J.; Basa, S.; et al. Joint Constraints on Galactic Diffuse Neutrino Emission from the ANTARES and IceCube Neutrino Telescopes. Astrophys. J. 2018, 868, L20. [CrossRef]

30. Aartsen, M.G.; Ackermann, M.; Adams, J.; Aguilar, J.A.; Ahlers, M.; Ahrens, M.; Alispach, C.; Andeen, K.; Anderson, T.; Ansseau, I.; et al. Time-integrated Neutrino Source Searches with 10 years of IceCube Data. Phys. Rev. Lett. 2020, $124,051103$. [CrossRef]

31. Aartsen, M.G.; Ackermann, M.; Adams, J.; Aguilar, J.A.; Ahlers, M.; Ahrens, M.; Altmann, D.; Anderson, T.; Archinger, M.; Arguelles, C.; et al. Searches for Time-dependent Neutrino Sources with IceCube Data from 2008 to 2012. Astrophys. J. 2015, 807, 46. [CrossRef] 
32. Acciari, V.A.; Ansoldi, S.; Antonelli, L.A.; Engels, A.A.; Baack, D.; Babić, A.; Banerjee, B.; de Almeida, U.B.; Barrio, J.A.; González, J.B.; et al. Constraints on Gamma-Ray and Neutrino Emission from NGC 1068 with the MAGIC Telescopes. Astrophys. J. 2019, 883, 135. [CrossRef]

33. Aartsen, M.G.; Ackermann, M.; Adams, J.; Aguilar, J.A.; Ahlers, M.; Ahrens, M.; Altmann, D.; Andeen, K.; Anderson, T.; Ansseau, I.; et al. The IceCube Realtime Alert System. Astropart. Phys. 2017, 92, 30-41. [CrossRef]

34. Santander, M.; Dorner, D.; Dumm, J.; Satalecka, K.; Schüssler, F. Searching for VHE gamma-ray emission associated with IceCube astrophysical neutrinos using FACT, H.E.S.S., MAGIC, and VERITAS. arXiv 2017, arXiv:1708.08945.

35. Barthelmy, S.D.; Butterworth, P.; Cline, T.L.; Gehrels, N.; Marshall, F.; Takeshima, T.; Connaughton, V.; Kippen, R.M.; Kouveliotou, C.; Robinson, C.R. The GRB coordinates network (GCN): A status report. AIP Conf. Proc. 1998, 428, 99-103. [CrossRef]

36. Smale, A.; Racusin, J.; Barthelmy, S.; McGlynn, T.; Cenko, B.; Schnittman, J.; Perkins, J.; Baker, J.; Singer, L.; Sheets, T.; et al. Time-domain Astronomy Coordination Hub (TACH). Bull. Am. Astron. Soc. 2020, 235, 107-115.

37. Rutledge, R.E. The Astronomer's Telegram: A Web-based Short-Notice Publication System for the Professional Astronomical Community. Publ. Astron. Soc. Pac. 1998, 110, 754-756. [CrossRef]

38. Dorner, D.; Arbet-Engels, A.; Baack, D.; Balbo, M.; Biland, A.; Bretz, T.; Buss, J.; Eisenberger, L.; Elsaesser, D.; Hildebrand, D.; et al. FACT-Highlights from more than Eight Years of Unbiased TeV Monitoring. In Proceedings of the 37th International Cosmic Ray Conference-PoS(ICRC2021), Berlin, Germany, 12-23 July 2021; Volume 395, p. 851. [CrossRef]

39. Ayala Solares, H.A. AMON Multimessenger Alerts: Past and Future. Galaxies 2019, 7, 19. [CrossRef]

40. Weisgarber, T. A new eye on the VHE transient universe with the HAWC online flare monitor. In Proceedings of the 6th International Symposium on High Energy Gamma-Ray Astronomy, Heidelberg, Germany, 11-15 July 2017; Volume 1792, p. 070009. [CrossRef]

41. Weisgarber, T.; Wisher, I.G. Blazar Alerts with the HAWC Online Flare Monitor. In Proceedings of the 34th International Cosmic Ray Conference (ICRC2015), Hague, The Netherlands, 30 July-6 August 2015; Volume 34, p. 798.

42. Ackermann, M.; Ajello, M.; Atwood, W.B.; Baldini, L.; Ballet, J.; Barbiellini, G.; Bastieri, D.; Gonzalez, J.B.; Bellazzini, R.; Bissaldi, E.; et al. 2FHL: The second catalog of hard fermi-lat sources. Astrophys. J. Suppl. Ser. 2016, 222, 5. [CrossRef]

43. Martinez, I.; HAWC Collaboration. Monitoring the TeV sky on hours long timescales with HAWC. In Proceedings of the 35th International Cosmic Ray Conference (ICRC2017), Busan, Korea, 12-20 July 2017; Volume 301, p. 656.

44. Telescope, L.; Aartsen, M.; Ackermann, M.; Adams, J.; Aguilar, J.A.; Ahlers, M.; Ahrens, M.; Al Samarai, I.; Altmann, D.; Andeen, K.; et al. Multimessenger observations of a flaring blazar coincident with high-energy neutrino IceCube-170922A. Science 2018, 361, eaat1378. [CrossRef]

45. Aartsen, M.G.; Ackermann, M.; Adams, J.; Aguilar, J.A.; Ahlers, M.; Ahrens, M.; Al Samarai, I.; Altmann, D.; Andeen, K.; Anderson, T.; et al. Differential limit on the extremely-high-energy cosmic neutrino flux in the presence of astrophysical background from nine years of IceCube data. Phys. Rev. D 2018, 98, 062003. [CrossRef]

46. Kronmueller, M.; Glauch, T. Application of Deep Neural Networks to Event Type Classification in IceCube. arXiv 2019, arXiv:1908.08763.

47. Huennefeld, M. Deep Learning in Physics exemplified by the Reconstruction of Muon-Neutrino Events in IceCube. PoS 2017, ICRC2017, 1057. [CrossRef]

48. Aartsen, M.G.; Ackermann, M.; Adams, J.; Aguilar, J.A.; Ahlers, M.; Ahrens, M.; Alispach, C.; Andeen, K.; Anderson, T.; Ansseau, I.; et al. Search for Sources of Astrophysical Neutrinos Using Seven Years of IceCube Cascade Events. Astrophys. J. 2019, 886, 12. [CrossRef]

49. Wood, J. An All-Sky Search for Bursts of Very High Energy Gamma Rays with HAWC. arXiv 2018, arXiv:1801.01550. .

50. Abbott, B.P.; Abbott, R.; Abbott, T.D.; Abraham, S.; Acernese, F.; Ackley, K.; Adams, C.; Adya, V.B.; Affeldt, C.; Agathos, M.; et al. Prospects for observing and localizing gravitational-wave transients with Advanced LIGO, Advanced Virgo and KAGRA. Living Rev. Relativ. 2020, 23, 3. [CrossRef]

51. Turley, C.F.; Fox, D.B.; Murase, K.; Falcone, A.; Barnaba, M.; Coutu, S.; Cowen, D.F.; Meszaros, P.; Mostafá, M.; Filippatos, G.; et al. Search for Blazar Flux-Correlated TeV Neutrinos in IceCube 40-String Data. Astrophys. J. 2016, 833, 117. [CrossRef]

52. Murase, K.; Turley, C.F. A Search for Cosmic Neutrino and Gamma-Ray Emitting Transients in 7.3 Years of ANTARES and Fermi LAT Data. Astrophys. J. 2019, 886, 98. [CrossRef]

53. Solares, H.A.; Coutu, S.; DeLaunay, J.J.; Fox, D.B.; Grégoire, T.; Keivani, A.; Krauß, F.; Mostafá, M.; Murase, K.; Turley, C.F.; et al. Multimessenger Gamma-Ray and Neutrino Coincidence Alerts Using HAWC and IceCube Subthreshold Data. Astrophys. J. 2021, 906, 63. [CrossRef]

54. Schüssler, F.; Ashkar, H.; Backes, M.; Egberts, K.; Brun, F.; Füssling, M.; Hoischen, C.; Lenain, J.P.; Lypova, I.; Ohm, S.; et al. H.E.S.S. searches for TeV gamma-rays associated to high-energy neutrinos. In Proceedings of the 36th International Cosmic Ray Conference (ICRC2019), Madison, WI, USA, 24 July-1 August 2019; Volume 36, p. 787.

55. Berti, A.; Bernardini, E.; Bhattacharyya, W.; Cortina, J.; Covino, S.; Dorner, D.; Espiñeira, E.D.S.; Fattorini, A.; Foffano, L.; Fukami, S.; et al. Following up Transient sources at Very High Energies with MAGIC. In Proceedings of the 36th International Cosmic Ray Conference (ICRC2019), Madison, WI, USA, 24 July-1 August 2019; Volume 36, p. 633.

56. Santander, M. Recent results from the VERITAS multi-messenger program. In Proceedings of the 36th International Cosmic Ray Conference (ICRC2019), Madison, WI, USA, 24 July-1 August 2019; Volume 36, p. 782. 
57. Dorner, D.; Adam, J.; Ahnen, L.M.; Baack, D.; Balbo, M.; Biland, A.; Blank, M.; Bretz, T.; Bruegge, K.; Bulinski, M.; et al. FACT-Highlights from more than Five Years of Unbiased Monitoring at TeV Energies. In Proceedings of the 35th International Cosmic Ray Conference (ICRC2017), Busan, Korea, 12-20 July 2017; Volume 301, p. 609.

58. Schüssler, F.; Backes, M.; Balzer, A.; Brun, F.; Füssling, M.; Hoischen, C.; Lenain, J.P.; Lorentz, M.; Lypova, I.; Ohm, S.; et al. The H.E.S.S. multi-messenger program: Searches for TeV gamma-ray emission associated with high-energy neutrinos. AIP Conf. Proc. 2017, 1792, 060006. [CrossRef]

59. Ageron, M.; Aguilar, J.A.; Al Samarai, I.; Albert, A.; André, M.; Anghinolfi, M.; Anton, G.; Anvar, S.; Ardid, M.; Jesus, A.A.; et al. The ANTARES telescope neutrino alert system. Astropart. Phys. 2012, 35, 530-536. [CrossRef]

60. Hoischen, C.; H.E.S.S. Collaboration. The H.E.S.S. transients alert system. In Proceedings of the New Era of Multi-Messenger Astrophysics (Asterics2019), Groningen, The Netherlands, 25-29 March 2019; Volume 357, p. 033.

61. Schüssler, F.; Backes, M.; Balzer, A.; Brun, F.; Füssling, M.; Hoischen, C.; Lenain, J.P.; Lypova, I.; Ohm, S.; Parsons, D.; et al. H.E.S.S. observations following multi-messenger alerts in real-time. In Proceedings of the 35th International Cosmic Ray Conference (ICRC2017), Busan, Korea, 12-20 July 2017; Volume 301, p. 653. [CrossRef]

62. Abbott, B.P.; Abbott, R.; Abbott, T.D.; Abernathy, M.R.; Acernese, F.; Ackley, K.; Adams, C.; Adams, T.; Addesso, P.; Adhikari, R.X.; et al. GW151226: Observation of Gravitational Waves from a 22-Solar-Mass Binary Black Hole Coalescence. Phys. Rev. Lett. 2016, 116, 241103. [CrossRef]

63. de Lotto, B.; Ansoldi, S.; Antonelli, A.; Berti, A.; Carosi, A.; Longo, F.; Stamerra, A. MAGIC electromagnetic follow-up of gravitational wave alerts. IAU Symp. 2016, 324, 287-290. [CrossRef]

64. Scientific, L.I.G.O.; Abbott, B.P.; Abbott, R.; Abbott, T.D.; Acernese, F.; Ackley, K.; Adams, C.; Adams, T.; Addesso, P.; Adhikari, R.X.; et al. GW170104: Observation of a 50-Solar-Mass Binary Black Hole Coalescence at Redshift 0.2. Phys. Rev. Lett. 2017, 118, 221101. [CrossRef]

65. Abbott, B.P.; Abbott, R.; Abbott, T.D.; Acernese, F.; Ackley, K.; Adams, C.; Adams, T.; Addesso, P.; Adhikari, R.X.; Adya, V.B.; et al. GW170814: A Three-Detector Observation of Gravitational Waves from a Binary Black Hole Coalescence. Phys. Rev. Lett. 2017, 119, 141101. [CrossRef]

66. Miceli, D.; Antonelli, L.A.; Gonzalez, J.B.; Berti, A.; Zeljka, B.; Covino, S.; Lotto, B.D.; Puppo, F.D.; Inoue, S.; Longo, F.; et al. Following up GW Alerts With MAGIC: The Third LIGO/Virgo Observation Run. arXiv 2019, arXiv:1909.03971.

67. Piron, F. Gamma-ray bursts at high and very high energies. Comptes Rendus Phys. 2016, 17, 617-631. [CrossRef]

68. Ahnen, M.L.; Ansoldi, S.; Antonelli, L.A.; Antoranz, P.; Arcaro, C.; Babic, A.; Banerjee, B.; Bangale, P.; De Almeida, U.B.; Barrio, J.A.; et al. Detection of very high energy gamma-ray emission from the gravitationally lensed blazar QSO B0218+357 with the MAGIC telescopes. Astron. Astrophys. 2016, 595, A98. [CrossRef]

69. Ahnen, M.L.; Ansoldi, S.; Antonelli, L.A.; Antoranz, P.; Babic, A.; Banerjee, B.; Bangale, P.; De Almeida, U.B.; Barrio, J.A.; Bednarek, W.; et al. Very High Energy $\gamma$-Rays from the Universe's Middle Age: Detection of the z $=0.940$ Blazar PKS $1441+25$ with MAGIC. Astrophys. J. Lett. 2015, 815, L23. [CrossRef]

70. Abeysekara, A.U.; Archambault, S.; Archer, A.; Aune, T.; Barnacka, A.; Benbow, W.; Bird, R.; Biteau, J.; Buckley, J.H.; Bugaev, V.; et al. Gamma-Rays from the Quasar PKS 1441+25: Story of an Escape. Astrophys. J. Lett. 2015, 815, L22. [CrossRef]

71. Veres, P.; Bhat, P.N.; Briggs, M.S.; Cleveland, W.H.; Hamburg, R.; Hui, C.M.; Mailyan, B.; Preece, R.D.; Roberts, O.J.; von Kienlin, A.; et al. Observation of inverse Compton emission from a long $\gamma$-ray burst. Nature 2019, 575, 459-463. [CrossRef]

72. Abdalla, H.; Adam, R.; Aharonian, F.; Benkhali, F.A.; Angüner, E.O.; Arakawa, M.; Arcaro, C.; Armand, C.; Ashkar, H.; Backes, M.; et al. A very-high-energy component deep in the $\gamma$-ray burst afterglow. Nature 2019, 575, 464-467. [CrossRef]

73. Abdalla, H.; Aharonian, F.; Benkhali, F.A.; Angüner, E.O.; Arcaro, C.; Armand, C.; Armstrong, T.; Ashkar, H.; Backes, M.; Baghmanyan, V.; et al. Revealing X-ray and gamma-ray temporal and spectral similarities in the GRB 190829A afterglow. Science 2021, 372, 1081-1085. [CrossRef]

74. Nava, L. Gamma-Ray Bursts at the highest energies. Galaxies. Universe, Accepted for publication in the special Issue "High-Energy Gamma-Ray Astronomy: Results on Fundamental Questions after 30 Years of Ground-Based Observations" (This Issue). 2021.

75. de Naurois, M.; H.E.S.S Collaboration. H.E.S.S. Follow-Up of IceCube-170922A. The Astronomer's Telegram. No. 10787. Available online: https:/ / www.astronomerstelegram.org/?read=10787 (accessed on 19 October 2021).

76. Mirzoyan, R.; MAGIC Collaboration. First-time Detection of VHE Gamma Rays by MAGIC from a Direction Consistent with the Recent Ehe Neutrino Event IceCube-170922A. The Astronomer's Telegram. No. 10817. Available online: https://www.astronomer stelegram.org/?read=10817 (accessed on 19 October 2021).

77. Ansoldi, S.; Antonelli, L.A.; Arcaro, C.; Baack, D.; Babić, A.; Banerjee, B.; Bangale, P.; De Almeida, U.B.; Barrio, J.A.; González, J.B.; et al. The Blazar TXS 0506+056 Associated with a High-energy Neutrino: Insights into Extragalactic Jets and Cosmic-Ray Acceleration. Astrophys. J. Lett. 2018, 863, L10. [CrossRef]

78. Abeysekara, A.U.; Archer, A.; Benbow, W.; Bird, R.; Brill, A.; Brose, R.; Buckley, J.H.; Christiansen, J.L.; Chromey, A.J.; Daniel, M.K.; et al. VERITAS observations of the BL Lac object TXS 0506+056. Astrophys. J. 2018, 861, L20. [CrossRef]

79. Cerruti, M.; Zech, A.; Boisson, C.; Emery, G.; Inoue, S.; Lenain, J.P. Leptohadronic single-zone models for the electromagnetic and neutrino emission of TXS 0506+056. MNRAS 2019, 483, L12-L16. [CrossRef]

80. Gao, S.; Fedynitch, A.; Winter, W.; Pohl, M. Modelling the coincident observation of a high-energy neutrino and a bright blazar flare. Nat. Astron. 2019, 3, 88-92. [CrossRef] 
81. Petropoulou, M.; Murase, K.; Santander, M.; Buson, S.; Tohuvavohu, A.; Kawamuro, T.; Vasilopoulos, G.; Negoro, H.; Ueda, Y.; Siegel, M.H.; et al. Multi-epoch Modeling of TXS 0506+056 and Implications for Long-term High-energy Neutrino Emission. Astrophys. J. 2020, 891, 115. [CrossRef]

82. Ros, E.; Kadler, M.; Perucho, M.; Boccardi, B.; Cao, H.M.; Giroletti, M.; Krauß, F.; Ojha, R. Apparent superluminal core expansion and limb brightening in the candidate neutrino blazar TXS 0506+056. Astron. Astrophys. 2020, 633, L1. [CrossRef]

83. Aartsen, M.; Ackermann, M.; Adams, J.; Aguilar, J.A.; Ahlers, M.; Ahrens, M.; Al Samarai, I.; Altmann, D.; Andeen, K.; Anderson, T.; et al. Neutrino emission from the direction of the blazar TXS 0506+056 prior to the IceCube-170922A alert. Science 2018, 361, 147-151. [CrossRef]

84. Paiano, S.; Falomo, R.; Treves, A.; Scarpa, R. The Redshift of the BL Lac Object TXS 0506+056. Astrophys. J. Lett. 2018, 854, L32. [CrossRef]

85. Benbow, W. Recent Results from VERITAS AGN Observations. In Proceedings of the 37th International Cosmic Ray Conference-PoS(ICRC2021), Berlin, Germany, 12-23 July 2021; Volume 395, p. 794. [CrossRef]

86. Satalecka, K.; Aniello, T.; Bernardini, E.; Bhattacharyya, W.; Cerruti, M.; D’Ammando, F.; Prandini, E.; Righi, C.; Sahakyan, N.; Viale, I.; et al. Multi-epoch monitoring of TXS 0506+056 with MAGIC and MWL partners. In Proceedings of the 37th International Cosmic Ray Conference-PoS(ICRC2021), Berlin, Germany, 12-23 July 2021; Volume 395, p. 875. [CrossRef]

87. Padovani, P.; Oikonomou, F.; Petropoulou, M.; Giommi, P.; Resconi, E. TXS 0506+056, the first cosmic neutrino source, is not a BL Lac. MNRAS 2019, 484, L104-L108. [CrossRef]

88. Abbott, B.P.; Abbott, R.; Abbott, T.D.; Acernese, F.; Ackley, K.; Adams, C.; Adams, T.; Addesso, P.; Adhikari, R.X.; Adya, V.B.; et al. Gravitational Waves and Gamma-Rays from a Binary Neutron Star Merger: GW170817 and GRB 170817A. Astrophys. J. 2017, 848, L13. [CrossRef]

89. Abdalla, H.; Abramowski, A.; Aharonian, F.; Benkhali, F.A.; Angüner, E.O.; Arakawa, M.; Arrieta, M.; Aubert, P.; Backes, M.; Balzer, A.; et al. TeV Gamma-Ray Observations of the Binary Neutron Star Merger GW170817 with H.E.S.S. Astrophys. J. Lett. 2017, 850, L22. [CrossRef]

90. Abdalla, H. Probing the Magnetic Field in the GW170817 Outflow Using H.E.S.S. Observations. Astrophys. J. 2020, 894 , L16. [CrossRef]

91. Reichherzer, P.; Schüssler, F.; Lefranc, V.; Yusafzai, A.; Alkan, A.K.; Ashkar, H.; Becker Tjus, J. Astro-COLIBRI-The COincidence LIBrary for Real-time Inquiry for multimessenger astrophysics. arXiv 2021, arXiv:2109.01672.

92. Benbow, W. Recent Results from VERITAS AGN Observations. arXiv 2021, arXiv:2108.05460.

93. Ayala Solares, H. Multimessenger NuEM Alerts with AMON. In Proceedings of the 37th International Cosmic Ray Conference-PoS(ICRC2021), Berlin, Germany, 12-23 July 2021; Volume 395, p. 958. [CrossRef]

94. Turley, C.F.; Fox, D.B.; Keivani, A.; DeLaunay, J.J.; Cowen, D.F.; Mostafá, M.; Ayala Solares, H.A.; Murase, K. A Coincidence Search for Cosmic Neutrino and Gamma-Ray Emitting Sources Using IceCube and Fermi-LAT Public Data. Astrophys. J. 2018, 863, 64. [CrossRef]

95. Tohuvavohu, A.; Kennea, J.A.; DeLaunay, J.; Palmer, D.M.; Cenko, S.B.; Barthelmy, S. Gamma-Ray Urgent Archiver for Novel Opportunities (GUANO): Swift/BAT Event Data Dumps on Demand to Enable Sensitive Subthreshold GRB Searches. Astrophys. J. 2020, 900, 35. [CrossRef]

96. Magee, R.; Fong, H.; Caudill, S.; Messick, C.; Cannon, K.; Godwin, P.; Hanna, C.; Kapadia, S.; Meacher, D.; Mohite, S.R.; et al. Sub-threshold Binary Neutron Star Search in Advanced LIGO's First Observing Run. Astrophys. J. 2019, 878, L17. [CrossRef]

97. Ben-Gal, I. Outlier Detection. In Data Mining and Knowledge Discovery Handbook; Maimon, O., Rokach, L., Eds.; Springer: Boston, MA, USA, 2005; pp. 131-146. [CrossRef]

98. Grégoire, T.; Ayala Solares, H.; Coutu, S.; Cowen, D.; DeLaunay, J.; Fox, D.B.; Keivani, A.; Krauss, F.; Mostafa, M.; Murase, K.; et al. Model independent search for transient multimessenger events with AMON using outlier detection methods. In Proceedings of the 37th International Cosmic Ray Conference-PoS(ICRC2021), Berlin, Germany, 12-23 July 2021; Volume 395, p. 934. [CrossRef]

99. Abreu, P. A search for ultra-high-energy photons at the Pierre Auger Observatory exploiting air-shower universality. In Proceedings of the 37th International Cosmic Ray Conference - PoS(ICRC2021), Berlin, Germany, 12-23 July 2021; Volume 395, p. 373. [CrossRef]

100. Acharya, B.S.; Agudo, I.; Al Samarai, I.; Alfaro, R.; Alfaro, J.; Alispach, C.; Batista, R.A.; Amans, J.P.; Amato, E.; Ambrosi, G.; et al. Science with the Cherenkov Telescope Array; The CTA Consortium; Published by World Scientific Publishing Co. Pte. Ltd.: Singapore, 2019. [CrossRef]

101. Fioretti, V.; Ribeiro, D.; Humensky, T.B.; Bulgarelli, A.; Maier, G.; Moralejo, A.; Nigro, C. The Cherenkov Telescope Array sensitivity to the transient sky. In Proceedings of the 36th International Cosmic Ray Conference-PoS(ICRC2019), Madison, WI, USA, 24 July-1 August 2019; Volume 358, p. 673. [CrossRef]

102. Di Piano, A.; Bulgarelli, A.; Fioretti, V.; Baroncelli, L.; Parmiggiani, N.; Longo, F.; Stamerra, A.; López-Oramas, A.; Stratta, G.; De Cesare, G. Detection methods for the Cherenkov Telescope Array at very-short exposure times. In Proceedings of the 37th International Cosmic Ray Conference-PoS(ICRC2021), Berlin, Germany, 12-23 July 2021; Volume 395, p. 694. [CrossRef]

103. Cao, Z.; Aharonian, F.A.; An, Q.; Bai, L.X.; Bai, Y.X.; Bao, Y.W.; Bastieri, D.; Bi, X.J.; Bi, Y.J.; Cai, H.; et al. Ultrahigh-energy photons up to 1.4 petaelectronvolts from $12 \gamma$-ray Galactic sources. Nature 2021, 594, 33-36. [CrossRef]

104. Cao, Z.; Aharonian, F.A.; An, Q.; Axikegu; Bai, L.X.; Bai, Y.X.; Bao, Y.W.; Bastieri, D.; Bi, X.J.; Bi, Y.J.; et al. PeV gamma-ray emission from the Crab Nebula. Science 2021. [CrossRef] 
105. Albert, A.; Alfaro, R.; Ashkar, H.; Alvarez, C.; Alvarez, J.; Arteaga-Velázquez, J.C.; Solares, H.A.; Arceo, R.; Bellido, J.A.; BenZvi, S.; et al. Science Case for a Wide Field-of-View Very-High-Energy Gamma-Ray Observatory in the Southern Hemisphere. arXiv 2019, arXiv:1902.08429.

106. Adrián-Martínez, S.; Ageron, M.; Aharonian, F.; Aiello, S.; Albert, A.; Ameli, F.; Anassontzis, E.; Andre, M.; Androulakis, G.; Anghinolfi, M.; et al. Letter of intent for KM3NeT 2.0. J. Phys. G Nucl. Phys. 2016, 43, 084001. [CrossRef]

107. Schumacher, L.J.; Huber, M.; Agostini, M.; Bustamante, M.; Oikonomou, F.; Resconi, E. PLEvM: A global and distributed monitoring system of high-energy astrophysical neutrinos. PoS 2021, ICRC2021, 1185. [CrossRef]

108. Aartsen, M.G.; Abbasi, R.; Ackermann, M.; Adams, J.; Aguilar, J.A.; Ahlers, M.; Ahrens, M.; Alispach, C.; Allison, P.; Amin, N.M.; et al. IceCube-Gen2: The Window to the Extreme Universe. arXiv 2020, arXiv:2008.04323.

109. Agostini, M.; Böhmer, M.; Bosma, J.; Clark, K.; Danninger, M.; Fruck, C.; Gernhäuser, R.; Gärtner, A.; Grant, D.; Henningsen, F.; et al. The Pacific Ocean Neutrino Experiment. Nat. Astron. 2020, 4, 913-915. [CrossRef]

110. Singer, L.P.; Price, L.R.; Farr, B.; Urban, A.L.; Pankow, C.; Vitale, S.; Veitch, J.; Farr, W.M.; Hanna, C.; Cannon, K.; et al. The first two years of electromagnetic follow-up with advanced ligo and virgo. Astrophys. J. 2014, 795, 105. [CrossRef]

111. Berry, C.P.L.; Mandel, I.; Middleton, H.; Singer, L.P.; Urban, A.L.; Vecchio, A.; Vitale, S.; Cannon, K.; Farr, B.; Farr, W.M.; et al. Parameter estimation for binary neutron-star coalescences with realistic noise during the advanced ligo era. Astrophys. J. 2015, 804, 114. [CrossRef]

112. Fairhurst, S. Localization of transient gravitational wave sources: Beyond triangulation. Class. Quantum Gravity 2018, $35,105002$. [CrossRef]

113. Chang, P.; Allen, G.; Anderson, W.; Bianco, F.B.; Bloom, J.S.; Brady, P.R.; Brazier, A.; Cenko, S.B.; Couch, S.M.; DeYoung, T.; et al. Cyberinfrastructure Requirements to Enhance Multi-messenger Astrophysics. arXiv 2019, arXiv:1903.04590.

114. European Open Science Cloud Future Project. Available online: https:/ / eoscfuture.eu (accessed on 19 October 2021). 